\title{
Oxidative Stress and Inflammation Interdependence in Multiple Sclerosis
}

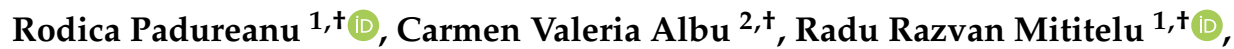 \\ Manuela Violeta Bacanoiu ${ }^{3,4,+}$, Anca Oana Docea ${ }^{5}$, Daniela Calina ${ }^{6}$, Vlad Padureanu ${ }^{7}$, \\ Gabriela Olaru ${ }^{3}$, Raluca Elena Sandu ${ }^{1,2}$, Ramona Denise Malin ${ }^{2}$ and Ana-Maria Buga $1, *$ (D) \\ 1 Department of Biochemistry, University of Medicine and Pharmacy of Craiova, 200349 Craiova, Romania; \\ zegheanurodica@yahoo.com (R.P.); razvanmititelu@rocketmail.com (R.R.M.); \\ raluv_2005@yahoo.com (R.E.S.) \\ 2 Department of Neurology, University of Medicine and Pharmacy of Craiova, 200349 Craiova, Romania; \\ carmenvaleriaalbu@yahoo.com (C.V.A.); ramona_denise@yahoo.com (R.D.M.) \\ 3 Department of Physical Therapy and Sports Medicine, University of Craiova, 200207 Craiova, Romania; \\ manuela_bacanoiu@yahoo.com (M.V.B.); olarugabriela246@yahoo.com (G.O.) \\ 4 Department of Laboratory Medicine, County Clinical Emergency Hospital of Craiova, \\ 200642 Craiova, Romania \\ 5 Department of Toxicology, University of Medicine and Pharmacy of Craiova, 200349 Craiova, Romania; \\ daoana00@gmail.com \\ 6 Department of Clinical Pharmacy, University of Medicine and Pharmacy of Craiova, 200349 Craiova, \\ Romania; calinadaniela@gmail.com \\ 7 Department of Internal Medicine, University of Medicine and Pharmacy of Craiova, 200349 Craiova, \\ Romania; vldpadureanu@yahoo.com \\ * Correspondence: anamaria.buga@umfcv.ro; Tel.: +40-0351-443-500 \\ + All these authors contributed equally to this work.
}

Received: 29 September 2019; Accepted: 28 October 2019; Published: 1 November 2019

\begin{abstract}
The study aims to explore the oxidative status related to inflammation in peripheral blood of stable relapsing-remitting multiple sclerosis (MS) patients with low disability. In this study, 31 people were included and divided into two groups: an MS group in which 16 relapsing-remitting MS patients with a low disability level (age $38.9 \pm 7.08$, EDSS median 2.5) were included and a control group that contains 15 healthy volunteers of similar age to the MS group. Thiobarbituric acid reactive substances (TBARS), protein carbonyl level (PCO), total antioxidant capacity (TAC) as oxidative stress markers, neutrophil/lymphocyte ratio (NLR), and erythrocyte sedimentation rate (ESR) were analyzed in the peripheral blood sample of the healthy and the MS patients to establish the oxidative stress/inflammatory level using conventional plasma markers. In this study, we showed that the pro-inflammatory status of the relapse-remitting stage of diseases can be easily and accurately appreciated by NLR. An increased NLR is associated with a decreased antioxidant capacity, even in the early stage of neuronal damage. Oxidative stress associated with inflammation aggravates the functional outcome, potentiates neuronal damage, and can accelerate the progression of the disease.
\end{abstract}

Keywords: multiple sclerosis; oxidative stress; neurodegeneration

\section{Introduction}

Oxidative stress involves a key pathway that contributes to many pathological processes including Multiple Sclerosis (MS) [1,2]. A balance between reactive oxygen species (ROS) production and antioxidant defence is crucial to prevent any structural damage to the CNS. The research studies in 
the last decade were focused on ROS action in CNS and its correlation with normal and pathological signaling pathways that target the neuronal cells [3,4].

It is well known that ROS production is necessary for neuronal cell stability, modulation of synaptic plasticity and brain functions (e.g., apoptosis, synaptic plasticity-related pathway including cognition, immune system) but the border between necessary and harmful processes is still very sensitive $[5,6]$.

An increased incidence of neurological and neurodegenerative diseases with aging can be a consequence of an imbalance between ROS production and antioxidant capacity of the brain tissue $[7,8]$. When the level of ROS increases in the cells, the capacity of the body to produce antioxidant molecules in a sufficient proportion is crucial to prevent the oxidative stress damage $[9,10]$.

Many pathological conditions of the CNS are associated with increased oxidative stress (e.g., Parkinson's disease, Alzheimer's disease) [11,12]. The pathophysiology of MS is still under debate. Many factors are potentially involved in the MS progression, including oxidative stress that promotes neurovascular unit injury and induces a pro-inflammatory state $[13,14]$.

In the early stage of MS, the immune system hyperactivation is the main pathological feature that promotes extensive damage of myelin by increasing the release of ROS. Later, in the chronic phase of MS, the main scenario involves structural and functional damage as a result of oxidative stress [15].

The neuronal tissue damage is a result of the inadaptive response of the antioxidant pathway in the early phase of the disease. MS progression can have a different clinical course related to the variability of adaptive mechanisms (relapsing, remitting or primary progressive courses). In this light, our hypothesis is that the disease progression can be decided in the early stages by promoting an enhanced and sustained antioxidant capacity of CNS tissue as early as possible. Furthermore, oxidative stress is associated with immune system hyperactivation through microglial cell activation. The activated microglial cells can release pro-oxidative signaling molecules (e.g., interleukin, cytokine) that can modulate the immune pathway, metabolic and/or apoptotic pathway in a time-dependent manner [9].

Since oxidative stress and inflammation correlate in a vicious cycle, recent studies have identified the biomarkers of oxidative stress and antioxidant capacity as potential tools to assess the inflammatory status in Multiple Sclerosis (MS) patients [16-18].

The aim of this study was to find potential peripheral biomarkers to show oxidative stress levels and antioxidant capacity in peripheral blood samples collected from MS patients in the remitting stage. Thiobarbituric acid reactive substances (TBARS), protein carbonyl level (PCO) and total antioxidant capacity (TAC) were analyzed in the peripheral blood sample of the healthy and MS patients with a low disability level (EDSS $\leq 4)$ to prove the oxidative stress level using conventional plasma markers. Also, our aim was to analyze the correlation between oxidative stress markers and inflammation in the subclinical stage of neuronal damage.

\section{Materials and Methods}

\subsection{Ethical Issues}

The study was approved by local institutional ethics committees of the University of Medicine and Pharmacy of Craiova (Registration No. 96) according to European Union Guidelines (Declaration of Helsinki). All participants gave written informed consent to be included in the study. Forty-one adult subjects were included in the study, 16 relapsing-remitting MS subjects (RRMS) (age $38.9 \pm 7.08$; first 10 years of disease progression and EDSS $\leq 4), 10$ secondary progressive MS subjects (SPMS) (more than eight years of disease progression and EDSS $>2$ ) and 15 adult healthy subjects with an appropriate median of age (age $37.1 \pm 11.2$ ). Exclusion criteria were the following: pregnancy, drug abuse, comorbidities that could increase the systemic inflammation (e.g., diabetes, metabolic syndrome), corticoids or non-steroidal anti-inflammatory drugs. All patients included in the MS group are non-smokers and received the first line-therapy (Glatiramel acetate or interferon beta). 


\subsection{Sample Collection and Handling}

The blood samples were collected in the morning after $12 \mathrm{~h}$ of fasting by an experimented phlebotomist using EDTA (ethylene-diamine-tetraacetic acid) tube collection, in order to prevent blood clotting. Plasma and red blood cell (RBC) fractions were separated by centrifugation at $3600 \mathrm{rpm}$ at $4{ }^{\circ} \mathrm{C}$ for $10 \mathrm{~min}$ (5417R Eppendorf centrifuge). The sediments were divided from plasma immediately after centrifugation and oxidative stress markers were analyzed in the plasma.

\subsection{Thiobarbituric Acid Reactive Substances Assay}

To analyze the lipid peroxidation level, we performed the thiobarbituric acid reactive substances (TBARS) plasma analysis using a UV spectrophotometric method $[19,20]$. The principles of procedure: malondialdehyde (MDA) is a common marker used to establish the oxidative stress index in human plasma. We analyzed the lipid peroxidation level by quantifying MDA concentration from deproteinized human plasma. Briefly, $0.1 \mathrm{~mL}$ human plasma was treated with $5 \%$ trichloroacetic acid (TCA) and $0.2 \mathrm{M}$ Tris- $\mathrm{HCl} \mathrm{pH}=4.7(v / v)$. After $10 \mathrm{~min}$ of incubation at room temperature (RT) the sample was mixed with $1 \mathrm{~mL}$ of $0.55 \mathrm{M}$ thiobarbituric acid (TBA) in $2 \mathrm{M}$ sodium sulphate, heated at $90^{\circ} \mathrm{C}$ for $45 \mathrm{~min}$ and cooled down in ice $[20,21]$. The optical density (OD) of the sample was measured at $530 \mathrm{~nm}$ using a UV-VIS spectrophotometer. The TBARS concentration was calculated using the molar extinction coefficient of MDA $\left(1.55 \times 105 \mathrm{M}^{-1} \mathrm{~cm}^{-1}\right)$. The results were expressed as TBARS $(\mu \mathrm{mol} / \mathrm{L})$.

\subsection{Protein Carbonyl Assay}

Protein carbonyls may be generated by irreversible oxidation of several amino acid side chains (Lysine, Arginine, Threonine, and Proline) from the carbonylation site in protein structure or by increased advanced glycation end products (AGE) production. The current successful method used to assess protein carbonyl (PCARB) concentration is a spectrophotometric assay using 2,4-dinitrophenylhydrazine (DNPH) $[20,22,23]$. The human plasma samples were mixed with $20 \%$ TCA $(v / v)$, incubated for $15 \mathrm{~min}$ on ice and separated by centrifugation for $5 \mathrm{~min} / 12,000 \mathrm{rpm}$ at $4{ }^{\circ} \mathrm{C}$. After centrifugation, the supernatant was discarded and the pellet was treated with $0.5 \mathrm{~mL} 10 \mathrm{mM}$ 2,4-dinitrophenylhydrazine (DNPH) in $2.5 \mathrm{M} \mathrm{HCl}$ for the test sample. The samples were incubated in the darkroom for $1 \mathrm{~h}$ with intermittent shaking every $15 \mathrm{~min}$. After incubation time, the samples were separated by centrifugation for $5 \mathrm{~min}$ at $12,000 \mathrm{rpm}$ at $4{ }^{\circ} \mathrm{C}$ and $10 \%$ TCA was added on the pellet, stored in ice for $10 \mathrm{~min}$, mixed by vortexing and separated by centrifugation for $5 \mathrm{~min}$ at 12,000 rpm at $4{ }^{\circ} \mathrm{C}$. The supernatant was removed and two washing steps were performed with $1 \mathrm{~mL}$ ethanol: ethyl acetate $(1: 1, v / v)$ to remove the excess of DNPH. The protein pellet was solved in $1 \mathrm{~mL}$ of $5 \mathrm{M}$ urea $(\mathrm{pH}$ $=2.3$ ) at $37^{\circ} \mathrm{C}$ for $10 \mathrm{~min}$ and separated by centrifugation for $5 \mathrm{~min}$ at $12,000 \mathrm{rpm}$ at $4{ }^{\circ} \mathrm{C}$. Finally, the OD samples were measured at $\lambda=375 \mathrm{~nm}$ using a UV-VIS spectrophotometer. The PCARB content was calculated based on the molar extinction factor of DNFH $\left(22,000 \mathrm{M}^{-1} \mathrm{~cm}^{-1}\right)$. The control sample with $2.5 \mathrm{M} \mathrm{HCL}$ was used during the protocol. The PCARB concentration was expressed as $\mathrm{nmol} / \mathrm{mg}$ of protein. Total protein concentration in the sample was assessed using Bradford reagent [24].

\subsection{Total Antioxidant Capacity (TAC) Assay}

TAC is usually used to assess the antioxidant status in human samples associated with diseases. TAC assessment show as a general capacity of the body to fight against oxidative stress by realizing antioxidant compounds. This capacity can be easily assessed in human plasma using a spectrophotometric method [20,25]. The human plasma samples diluted 1: 25 in $1 \times$ Phosphate Buffer Saline (PBS, $\mathrm{pH}=7.4$ ) were mixed with $0.1 \mathrm{mM} 2,2$ diphenyl-1-picrylhydrazyl reagent $(v / v)$ and incubated in a dark room for $30 \mathrm{~min}$. After incubation time, the samples were separated by centrifugation for $3 \mathrm{~min}$ at $14,000 \mathrm{rpm}$ and OD was read at $\lambda=520 \mathrm{~nm}$ using a UV-VIS spectrophotometer. TAC was expressed as mmol DPPH/L. 


\subsection{Neutrophil/Lymphocyte Ratio}

Neutrophil and lymphocyte were counted in peripheral blood obtained by standard venipuncture (EDTA collection tube) using an automatic flow cytometry analyzer (CELL-DYN Ruby System, Abbott Diagnostics). The neutrophil/lymphocyte ratio (NLR) was calculated by dividing the neutrophil number by lymphocyte number [26].

\subsection{Statistical Analysis}

Data were analyzed using GraphPad Prism 5.0 software. The comparison of oxidative stress markers between groups was performed using unpaired non-parametric Mann-Whitney $t$-test. Non-parametric Pearson's was calculated to test the correlation between biochemical and clinical variables (EDSS). $p$ values under $0.05(p \leq 0.05)$ were selected as significant changes. The data are calculated as mean \pm standard error of the mean (SEM).

\section{Results}

\subsection{Clinical and Biological Characteristics}

The MS patients included in this study are in the relapsing-remitting stage of diseases (MS group 1) with a low disability level (EDSS $=1-4$ ) and secondary progressive MS subjects (more than eight years of disease progression and EDSS $\geq 2$ ). The differences between the MS groups' and the healthy control group's biological variables, age, disability score (EDSS) are presented in Table 1.

Table 1. Demographic data of multiple sclerosis (MS) and healthy control group.

\begin{tabular}{ccccc}
\hline Groups & $\begin{array}{c}\text { Age Mean } \pm \text { SD } \\
\text { Age Range }\end{array}$ & Sex & $\begin{array}{c}\text { Disease } \\
\text { Progression Mean } \\
\text { (Range) }\end{array}$ & EDSS Mean \\
\hline MS group 1 1 & $38.9 \pm 7.08(28-50)$ & 13 Female 3 Male & 7 years (0-10) & $2.5(1-4)$ \\
MS group 2 $^{2}$ & $54 \pm 9.23(47-69)$ & 10 Female & 13 years (8-21) & $5(2-7)$ \\
Control group & $37.1 \pm 11.2(26-49)$ & 12 Female 3 Male & - & - \\
\hline${ }^{1}$ RRMS (relapsing-remitting multiple sclerosis) group with low disability level (EDSS $\left.=1-4\right) ;{ }^{2}$ SPMS (secondary \\
progressive multiple sclerosis) group (EDSS $=2-4)$.
\end{tabular}

Hyperactivation of the immune system response can be monitored using a blood test marker like erythrocyte sedimentation rate (ESR) and neutrophil/lymphocyte ratio (NLR) (Table 2).

Table 2. Biological markers of inflammation status in MS and healthy control groups.

\begin{tabular}{ccc}
\hline Groups & ESR Mean \pm SD & NLR Mean \pm SD \\
\hline MS group 1 & $24.5 \pm 9.15^{1}$ & $2.11 \pm 0.11^{1}$ \\
MS group 2 & $35.4 \pm 16.5$ & $2.8 \pm 0.71^{1}$ \\
Control group & $12.79 \pm 1.84$ & $1.61 \pm 0.08$ \\
\hline \multicolumn{2}{c}{${ }^{1}$ These parameters were significant higher. }
\end{tabular}

\subsection{Clinical and Biological Characteristics}

\subsubsection{Neurophil/Lymphocyte Ratio}

Currently, NLR was described as a new biomarker associated with inflammation that is accurate and easy to perform. NLR was significantly higher in MS group 1 than in the control group $(p=0.0025)$ as presented in Figure 1a. Also, we found that NLR was significant higher in MS group 2 vs MS group $1(p=0.001)$ as shown in Figure 1b. 
NLR

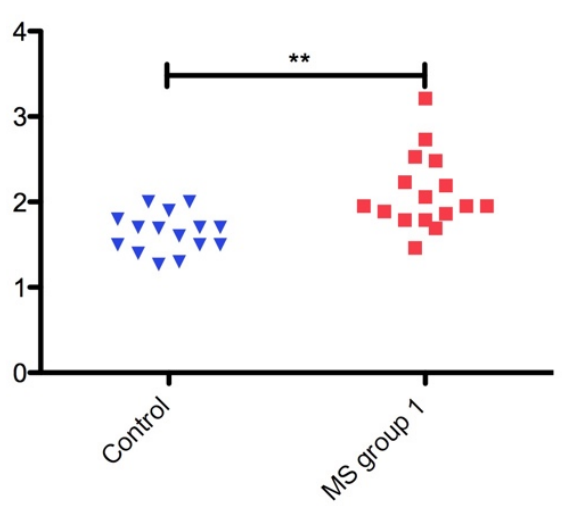

(a)
NLR

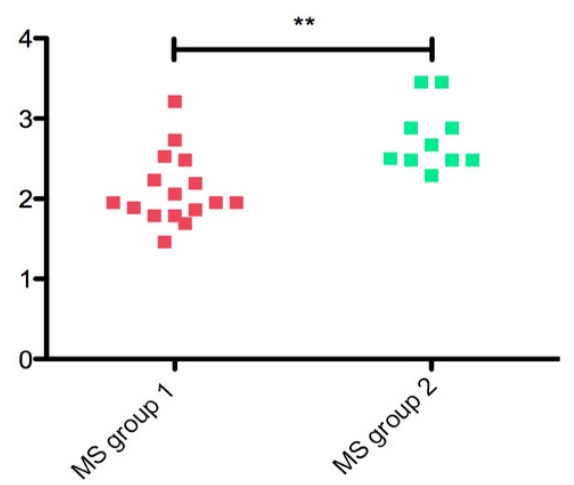

(b)

Figure 1. NLR in MS groups and control group: (a) scatter plot of NLR in control group and MS group $1\left(p=0.0025^{* *}\right)$; (b) scatter plot of NLR in MS group 1 and MS group $2\left(p=0.0010^{* *}\right)$.

\subsubsection{Oxidative Stress Markers vs. Total Antioxidant Capacity (TAC) in MS Patients}

The oxidative stress marker in the plasma was significantly increased in RRMS patients compared with the control group. TBARS $\left(p=0.0001^{* * *}\right)$ and PCARB $\left(p=0.0007^{* * *}\right)$ were significantly higher in MS patients than those in the control group (Figure 2)

TBARS

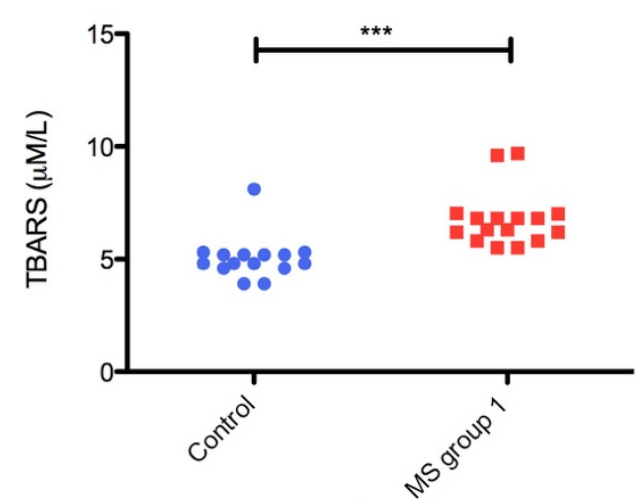

(a)
PCARB

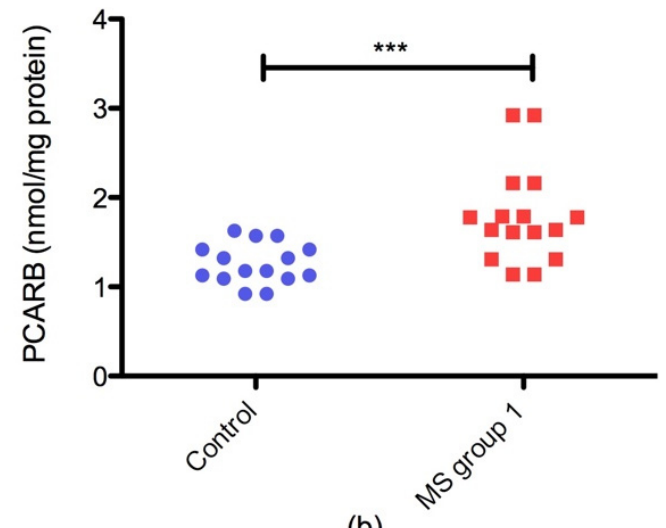

(b)

Figure 2. Plasmatic oxidative stress markers in RRMS groupvs control group: (a) scatter plot of TBARS levels; (b) scatter plot of PCARB (protein carbonyl) level in control group and RRMS group.

In contrast, total antioxidant capacity (TAC) was significantly decreased $\left(p=0.04^{*}\right)$ in RRMS patients as compared to the healthy control group (Figure 3). 


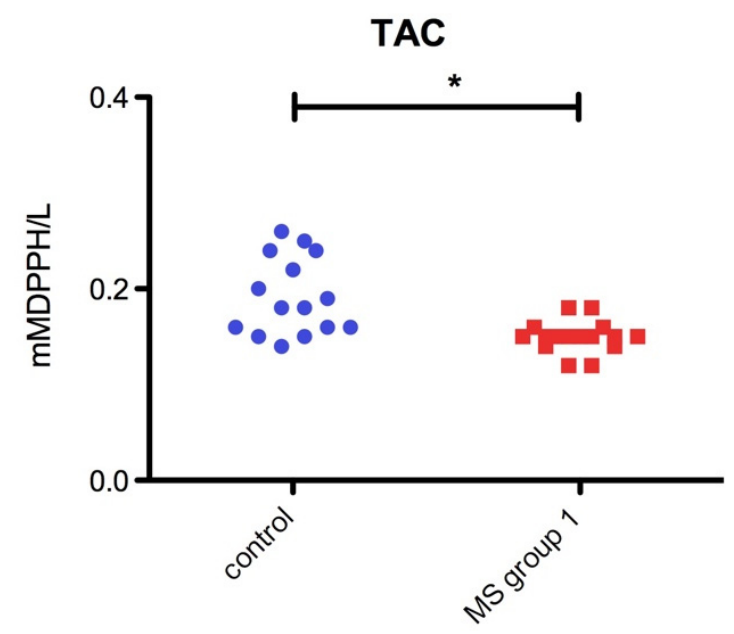

Figure 3. Bar plot (mean with SEM) of plasmatic TAC level in Control group and RRMS group $\left(p=0.04^{*}\right)$.

\subsubsection{Correlation of Oxidative Stress Status with Disability Stage (EDSS) and Inflammatory Status}

Interestingly, we found a significant negative correlation between TAC level and NLR $(p=0.02)$ in group 1 MS patients' plasma (Figure 4c) and between EDSS and TAC in group 2 MS patients (Figure 4f). We did not find any significant correlation between EDSS and NLR $(p=0.05)$ or ESR and NLR ( $p=0.79)$ in MS patients groups. Also, oxidative stress markers (TBARS and PCARB) did not correlate significantly with NLR in the MS groups (Figure 4).

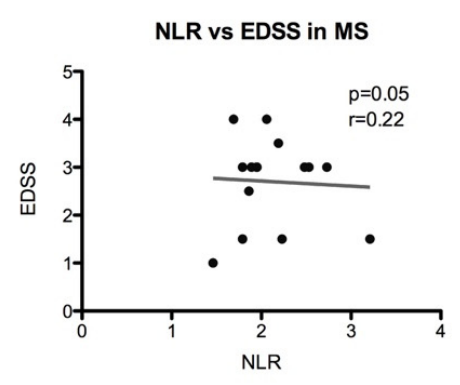

(a)

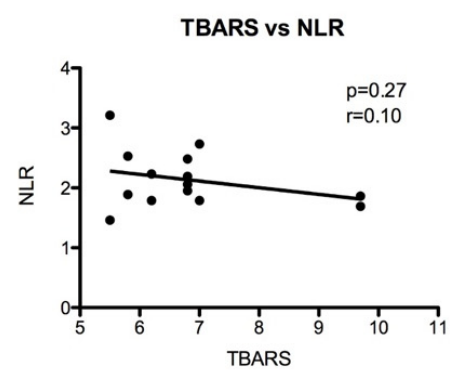

(d)

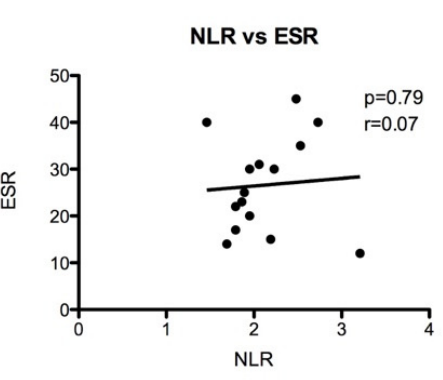

(b)

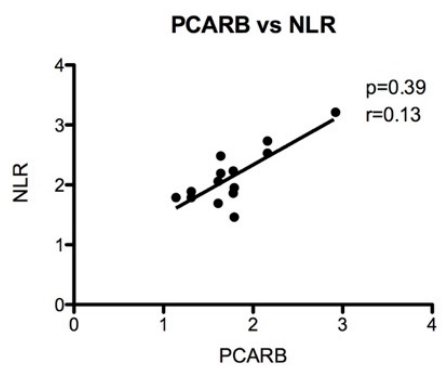

(e)

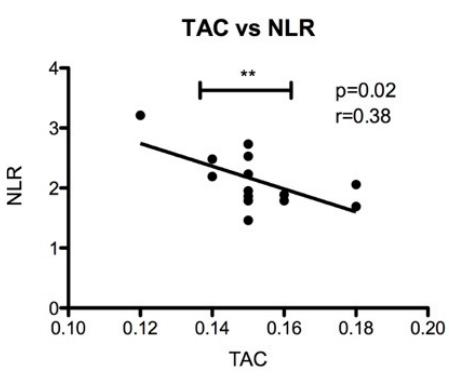

(c)

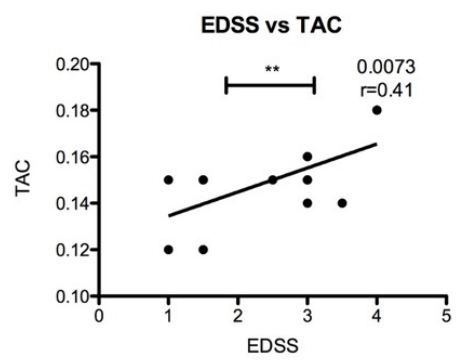

(f)

Figure 4. Correlation between inflammation marker NLR, oxidative stress status in plasma and disability stage (EDSS): (a) NLR correlation with EDSS $(p=0.05)$; (b) correlation between NLR and $\operatorname{ESR}(p=0.79) ;(\mathbf{c})$ TAC and NLR correlation $(p=0.02 * *)$; (d) TBARS and NLR correlation $(p=0.27)$;

(e) PCARB with NLR correlation $(p=0.39)$; (f) EDSS and TAC correlation in MS group $2\left(p=0.0073^{* *}\right)$.

Also, we did not find a significant correlation of EDSS with PCARB and TBARS levels in MS group 2 patients. 


\subsubsection{Receiver Operating Characteristic Curve (ROC Analysis)}

We performed a receiver operating characteristic (ROC) analysis of the inflammation marker and the oxidative stress marker in the MS patient groups compared with the healthy control group. Interestingly, we found TBARS as the most specific marker of oxidative stress with the highest area under the curve $(A U C)$ value $(A U C=0.94)$ followed by PCARB level $(A U C=0.86)$. NLR has a moderate specificity (AUC $=0.82$ ) to MS diseases in the early stage. In contrast, TAC has low specificity $(\mathrm{AUC}=0.71)$ in this stage of diseases (Figure 5$)$.

\section{ROC of TAC}

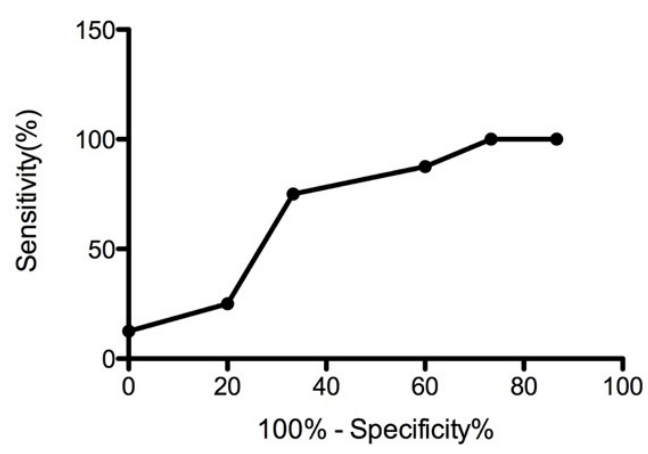

AUC: $0.71(95 \% \mathrm{Cl}: 0.53-0.9)$

(a)

ROC of TBARS

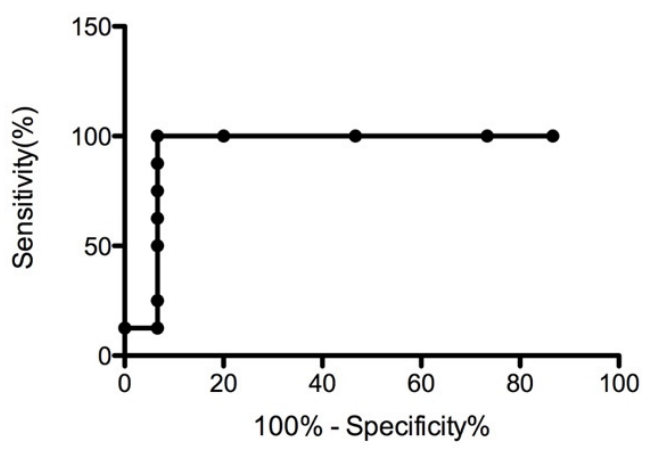

AUC: 0.94 ( 95\%Cl: 0.85-1.05)

(c)

\section{ROC of NLR}

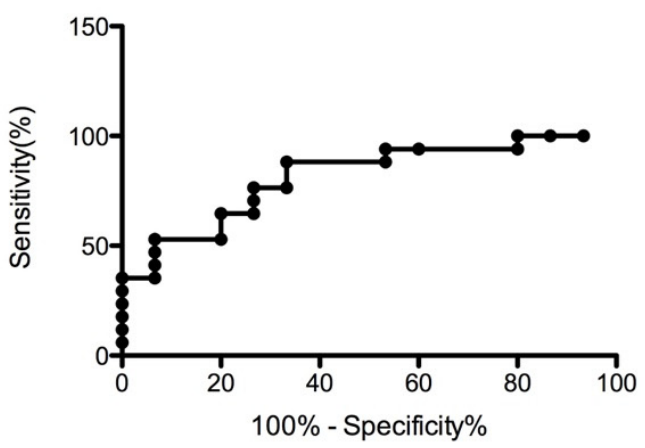

AUC: 0.82 ( $95 \% \mathrm{Cl}: 0.67-0.96)$

(b)

ROC of PCARB

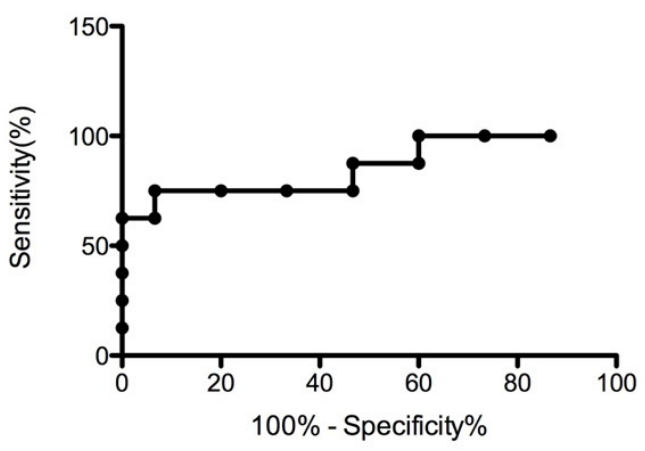

AUC: 0.86 (95\%Cl: 0.73-0.99)

(d)

Figure 5. ROC analysis of inflammation marker NLR and oxidative stress marker in plasma: (a) TAC assay characteristics in MS (AUC $=0.71$ ); (b) NLR characteristics (AUC $=0.82)$; (c) TBARS specificity $(\mathrm{AUC}=0.94) ;(\mathbf{d})$ PCARB assay performance $(\mathrm{AUC}=0.86)$.

Being a graphical plot that illustrates the diagnostic ability of a binary classifier system, the ROC curve is actually the sensitivity as a function of fall-out. Considering the diagnosis as the output and any different parameter as the single input for the classifier we can use the area under the ROC curve as a measure for the ration between the parameter taken into consideration and the diagnosis.

A good interpretation of the graphical representation uses the following rule: one point in space is better than another if it is situated to the northwest of the square. Technically, if the true positive rate is high and the false positive rate is low then the prediction is better. We can see from Figure 5, that the points are above the line of no-discrimination implying a good classification. In terms of the value 
of the AUC we can see that for TBARS the classification is very good, AUC $=0.94$, for the NLR and PCARB the classification is good, $\mathrm{AUC}=0.82$, and $\mathrm{AUC}=0.86$ respectively, whereas for the TAC the classification is fair, $\mathrm{AUC}=0.71$.

\section{Discussion}

To our knowledge, this is the first study to evaluate the NLR and oxidative stress association in MS patients. In this study, we found that the MS patients display a significant increase of oxidative stress markers in plasma (TBARS and PCARB) suggesting that oxidative stress induction started in the RRMS stage with a low level of irreversible neuronal damage. This induction is due to an imbalance between oxidative stress triggers and the capacity of the body to neutralize ROS as shown by a significant decrease of the TAC assay in plasma of MS patients. In the present work, we employed a TAC assay in plasma, to test the general oxidative stress protection capacity of the body in response to an increased ROS level. TAC includes all antioxidant compounds (enzyme or other molecules) that protect the body, not only at the systemic level but also CNS level [27-29].

Oxidative stress affects endogenous antioxidant defense capacity leading to neuronal damage [30,31]. Also, endogenous sources of ROS include inflammation, and ROS can act as a secondary brain injury after long-term pro-inflammatory status in a vicious cycle. In line with other research studies [18,32], we found a decreased antioxidant capacity that exposes the brain tissue to neuronal damage. Also, we showed that the TAC marker in plasma is significantly correlated with the inflammation marker NLR in RRMS patients and can be useful in the clinical management of diseases. There are still controversial findings of oxidative stress involvement in neuronal damage in the RRMS stage of diseases. Some studies suggest the importance of ROS action in the progressive stage of MS, and not in the pro-inflammatory stage $[15,33]$. These studies prove that the antioxidant defense system is affected in RRMS and is correlated with inflammatory status. Also, we found a low specificity of TAC assay alone in RRMS diseases. In contrast, TAC is significantly correlated with EDSS in secondary progressive MS patients.

NLR was described before to be an independent predictive marker of MS disease activity [26]. Studies before proved that the NLR is correlated with poor outcomes in many other diseases (e.g. acute myocardial infarction, cancers, autoimmune diseases, etc.) [34]. Also, neutrophils granule contains enzyme-like lysozyme or lactoferrin which can be markers of organ-specific inflammation [35]. However, only limited studies described NLR association in MS diseases [36,37] and it is still a growing interest aiming to establish potential serological markers associated with a local and systemic inflammatory status that are cost-effective and minimally invasive. We found that NLR did not correlate with EDSS and has a medium specificity alone in MS diseases, but these findings should be validated on a large cohort.

The brain tissue is highly sensitive to ROS action due to the increased consumption of oxygen and a high concentration of polyunsaturated fatty acids that are sensitive to lipid peroxidation $[29,38]$. In this light, the lipid peroxidation indicator in plasma was assessed using the detection of TBARS released by oxidative processes of polyunsaturated fatty acids (PUFA). An increased level of TBARS was found in RRMS patients but did not correlate with inflammation status. Interestingly, the TBARS assay displayed the highest specificity in the RRMS and can be a useful tool in MS disease management. In contrast, we found a significant correlation of EDSS with TAC in SPMS patients and we did not find a significant correlation with PCARB and TBARS in the stage of this disease. A possible explanation is due to the limited number of patients included in this study.

In order to support these findings, another oxidative stress marker PCARB was significantly increased in MS patients. Increased PCARB level was described before in neurodegenerative diseases but the link between PCARB level, inflammation and disease outcome is still under debate. PCARB is a useful tool to assess the irreversible changes in protein structure and considered a hallmark of increased ROS action and maybe a key player in MS disease progression [39,40]. In this study, we 
found that PCARB has an increased specificity (after TBARS) in MS diseases and did not correlate with NLR.

\section{Conclusions}

Enhanced protection of CNS by a sustained antioxidant capacity plays a crucial role in MS patient management to limit the irreversible neuronal damage. An increased level of oxidative stress markers and decreased level of TAC starting from subclinical neuronal damage was found. Also, in this study we showed that: (1) the pro-inflammatory status of the relapse-remitting stage of diseases can be easily and accurately appreciated by NLR and (2) an increased NLR is associated with a decreased antioxidant capacity, even in the subclinical stage of neuronal damage that affects the functional outcome (EDSS $=2.5$ ), potentiates neuronal damage and can accelerate the progression of the disease; (3) TBARS assay displayed the highest specificity in the RRMS patients and can be a useful tool in MS diseases management.

These show that oxidative stress is a key factor, together with the inflammatory state, that can contribute to disease progression. An increased oxidative stress level with a proinflammatory status can be a preclinical sign applying for proper intervention to support the antioxidant homeostasis. The NLR is an easy-to-perform biomarker that is accurate and can show the proinflammatory status of MS patients. In our opinion, a multimodal approach is needed to be added to MS management starting in the first 10 years of disease progression in order to improve the functional outcome. However, our study is limited to a small number of patients and has to be further explored in a large cohort.

Author Contributions: Conceptualization, R.P., C.V.A., R.R.M., M.V.B., V.P., A.O.D., D.C., R.E.S. and A.-M.B.; methodology, A.O.D., M.V.B., R.P., C.V.A., R.R.M., D.C. and G.O.; formal analysis, A.-M.B., R.P., C.V.A., R.R.M., R.D.M. and M.V.B.; data curation, R.D.M., S.R.E., R.P., C.V.A., R.R.M., M.V.B., V.P. and A.-M.B.; writing-A.-M.B., R.P., C.V.A., R.R.M., M.V.B., V.P., G.O.; writing-review and editing, A.O.D., D.C. and R.E.S.; supervision, A.-M.B. and A.O.D.

Acknowledgments: This study is part of the PhD theses of Rodica Padureanu from the University of Medicine and Pharmacy of Craiova, Craiova, Romania. We are thankful to Loredana Colhon for technical support.

Conflicts of Interest: The authors declare no conflict of interest.

\section{References}

1. D'evoli, L.; Morroni, F.; Lombardi-Boccia, G.; Lucarini, M.; Hrelia, P.; Cantelli-Forti, G.; Tarozzi, A. Red chicory (Cichorium intybus L. cultivar) as a potential source of antioxidant anthocyanins for intestinal health. Oxidative Med. Cell. Longev. 2013, 2013, 704310. [CrossRef]

2. Maffei, F.; Tarozzi, A.; Carbone, F.; Marchesi, A.; Hrelia, S.; Angeloni, C.; Forti, G.C.; Hrelia, P. Relevance of apple consumption for protection against oxidative damage induced by hydrogen peroxide in human lymphocytes. Br. J. Nutr. 2007, 97, 921-927. [CrossRef] [PubMed]

3. Serrano, F.; Chang, A.; Hernandez, C.; Pautler, R.G.; Sweatt, J.D.; Klann, E. NADPH oxidase mediates beta-amyloid peptide-induced activation of ERK in hippocampal organotypic cultures. Mol. Brain 2009, 2, 31. [CrossRef] [PubMed]

4. Massaad, C.A.; Klann, E. Reactive oxygen species in the regulation of synaptic plasticity and memory. Antioxid. Redox Signal. 2011, 14, 2013-2054. [CrossRef]

5. Kemmerling, U.; Muñoz, P.; Müller, M.; Sánchez, G.; Aylwin, M.L.; Klann, E.; Carrasco, M.A.; Hidalgo, C. Calcium release by ryanodine receptors mediates hydrogen peroxide-induced activation of ERK and CREB phosphorylation in N2a cells and hippocampal neurons. Cell Calcium 2007, 41, 491-502. [CrossRef]

6. Betzen, C.; White, R.; Zehendner, C.M.; Pietrowski, E.; Bender, B.; Luhmann, H.J.; Kuhlmann, C.R. Oxidative stress upregulates the NMDA receptor on cerebrovascular endothelium. Free Radic. Biol. Med. 2009, 47, 1212-1220. [CrossRef]

7. Grimm, A.; Eckert, A. Brain aging and neurodegeneration: From a mitochondrial point of view. J. Neurochem. 2017, 143, 418-431. [CrossRef]

8. Singh, A.; Kukreti, R.; Saso, L.; Kukreti, S. Oxidative Stress: A Key Modulator in Neurodegenerative Diseases. Molecules 2019, 24, 1583. [CrossRef] 
9. Rojo, A.I.; McBean, G.; Cindric, M.; Egea, J.; López, M.G.; Rada, P.; Zarkovic, N.; Cuadrado, A. Redox control of microglial function: Molecular mechanisms and functional significance. Antioxid. Redox Signal. 2014, 21, 1766-1801. [CrossRef]

10. Moretti, M.; Fraga, D.B.; Rodrigues, A. Preventive and therapeutic potential of ascorbic acid in neurodegenerative diseases. CNS Neurosci. Ther. 2017, 23, 921-929. [CrossRef]

11. Trist, B.G.; Hare, D.J.; Double, K.L. Oxidative stress in the aging substantia nigra and the etiology of Parkinson's disease. Aging Cell 2019, e13031. [CrossRef] [PubMed]

12. Ruthirakuhan, M.; Herrmann, N.; Andreazza, A.C.; Verhoeff, N.; Gallagher, D.; Black, S.E.; Kiss, A.; Lanctôt, K.L. Agitation, Oxidative Stress, and Cytokines in Alzheimer Disease: Biomarker Analyses from a Clinical Trial With Nabilone for Agitation. J. Geriatr. Psychiatry Neurol. 2019, 891988719874118. [CrossRef] [PubMed]

13. Carvalho, C.; Moreira, P.I. Oxidative Stress: A Major Player in Cerebrovascular Alterations Associated to Neurodegenerative Events. Front. Physiol. 2018, 9, 806. [CrossRef] [PubMed]

14. Lutton, E.M.; Farney, S.K.; Andrews, A.M.; Shuvaev, V.V.; Chuang, G.Y.; Muzykantov, V.R.; Ramirez, S.H. Endothelial Targeted Strategies to Combat Oxidative Stress: Improving Outcomes in Traumatic Brain Injury. Front. Neurol. 2019, 10, 582. [CrossRef] [PubMed]

15. Miller, E.; Wachowicz, B.; Majsterek, I. Advances in antioxidative therapy of multiple sclerosis. Curr. Med. Chem. 2013, 20, 4720-4730. [CrossRef]

16. Miller, E.; Walczak, A.; Saluk, J.; Ponczek, M.B.; Majsterek, I. Oxidative modification of patient's plasma proteins and its role in pathogenesis of multiple sclerosis. Clin. Biochem. 2012, 45, 26-30. [CrossRef]

17. Oliveira, S.R.; Kallaur, A.P.; Simão, A.N.C.; Morimoto, H.K.; Lopes, J.; Panis, C.; Petenucci, D.L.; da Silva, E.; Cecchini, R.; Kaimen-Maciel, D.R.; et al. Oxidative stress in multiple sclerosis patients in clinical remission: Association with the expanded disability status scale. J. Neurol. Sci. 2012, 321, 49-53. [CrossRef]

18. Siotto, M.; Filippi, M.M.; Simonelli, I.; Landi, D.; Ghazaryan, A.; Vollaro, S.; Ventriglia, M.; Pasqualetti, P.; Rongioletti, M.C.A.; Squitti, R.; et al. Oxidative Stress Related to Iron Metabolism in Relapsing Remitting Multiple Sclerosis Patients with Low Disability. Front. Neurosci. 2019, 13, 86. [CrossRef]

19. Liu, J.; Yeo, H.C.; Daniger, S.J.; Ames, B.N. Assay of aldehydes from lipid peroxidation: Gas chromatography mass spectrometry compared with thiobarbituric acid. Anal. Biochem. 1997, 245, 161-166. [CrossRef]

20. Spanidis, Y.; Goutzourelas, N.; Stagos, D.; Mpesios, A.; Priftis, A.; Bar-Or, D.; Spandidos, D.A.; Tsatsakis, A.M.; Leon, G.; Kouretas, D. Variations in oxidative stress markers in elite basketball players at the beginning and end of a season. Exp. Ther. Med. 2016, 11, 147-153. [CrossRef]

21. Keles, M.S.; Taysi, S.; Sen, N.; Aksoy, H.; Akçay, F. Effect of corticosteroid therapy on serum and CSF malondialdehyde and antioxidant proteins in multiple sclerosis. Can. J. Neurol. Sci. 2001, 28, 141-143. [CrossRef] [PubMed]

22. Patsoukis, N.; Papapostolou, I.; Zervoudakis, G.; Georgiou, C.D.; Matsokis, N.A.; Panagopoulos, N.T. Thiol redox state and oxidative stress in midbrain and striatum of weaver mutant mice, a genetic model of nigrostriatal dopamine deficiency. Neurosci. Lett. 2005, 376, 24-28. [CrossRef] [PubMed]

23. Colombo, G.; Clerici, M.; Garavaglia, M.E.; Giustarini, D.; Rossi, R.; Milzani, A.; Dalle-Donne, I. A step-by-step protocol for assaying protein carbonylation in biological samples. J. Chromatogr. B Anal. Technol. Biomed. Life Sci. 2016, 1019, 178-190. [CrossRef] [PubMed]

24. Bradford, M. A Rapid and Sensitive Method for the Quantification of Microgram Quantities of Protein Utilizing the Principle of Protein-Dye Binding. Anal. Biochem. 1976, 72, 248-254. [CrossRef]

25. Janaszewska, A.; Bartosz, G. Assay of total antioxidant capacity: Comparison of four methods as applied to human blood plasma. Scand. J. Clin. Lab. Investig. 2002, 62, 231-236. [CrossRef] [PubMed]

26. Demirci, S.; Demirci, S.; Kutluhan, S.; Koyuncuoglu, H.R.; Yurekli, V.A. The clinical significance of the neutrophil-to-lymphocyte ratio in multiple sclerosis. Int. J. Neurosci. 2016, 126, 700-706. [CrossRef]

27. Enciu, A.M.; Gherghiceanu, M.; Popescu, B.O. Triggers and effectors of oxidative stress at blood-brain barrier level: Relevance for brain ageing and neurodegeneration. Oxidative Med. Cell. Longev. 2013, 2013, 297512. [CrossRef]

28. Falkowski, M.; Maciejczyk, M.; Koprowicz, T.; Mikołuć, B.; Milewska, A.; Zalewska, A.; Car, H. Whey Protein Concentrate WPC-80 Improves Antioxidant Defense Systems in the Salivary Glands of 14-Month Wistar Rats. Nutrients 2018, 10, 782. [CrossRef] 
29. Maciejczyk, M.; Żebrowska, E.; Zalewska, A.; Chabowski, A. Redox Balance, Antioxidant Defense, and Oxidative Damage in the Hypothalamus and Cerebr Cortex of Rats with High Fat Diet-Induced Insulin Resistance. Oxidative Med. Cell. Longev. 2018, 2018, 6940515. [CrossRef]

30. Frati, A.; Cerretani, D.; Fiaschi, A.I.; Frati, P.; Gatto, V.; La Russa, R.; Pesce, A.; Pinchi, E.; Santurro, A.; Fraschetti, F.; et al. Diffuse Axonal Injury and Oxidative Stress: A Comprehensive Review. Int. J. Mol. Sci. 2017, 18, 2600. [CrossRef]

31. Neri, M.; Büttner, A.; Fineschi, V. Brain Injury due to Mechanical Trauma and Ischemic-Hypoxic Insult: Biomarkers of Brain Injury and Oxidative Stress. Oxidative Med. Cell. Longev. 2017, 2017, 8923472. [CrossRef] [PubMed]

32. Tasset, I.; Agüera, E.; Sánchez-López, F.; Feijóo, M.; Giraldo, A.I.; Cruz, A.H.; Gascón, F.; Túnez, I. Peripheral oxidative stress in relapsing-remitting multiple sclerosis. Clin. Biochem. 2012, 45, 440-444. [CrossRef] [PubMed]

33. Choi, I.Y.; Lee, P.; Adany, P.; Hughes, A.J.; Belliston, S.; Denney, D.R.; Lynch, S.G. In vivo evidence of oxidative stress in brains of patients with progressive multiple sclerosis. Mult. Scler. J. 2018, 24, 1029-1038. [CrossRef] [PubMed]

34. Chen, J.; Hong, D.; Zhai, Y.; Shen, P. Meta-analysis of associations between neutrophil-to-lymphocyte ratio and prognosis of gastric cancer. World J. Surg. Oncol. 2015, 13, 122. [CrossRef]

35. La Russa, R.; Maiese, A.; Viola, R.V.; De Matteis, A.; Pinchi, E.; Frati, P.; Fineschi, V. Searching for highly sensitive and specific biomarkers for sepsis: State-of-the-art in post-mortem diagnosis of sepsis through immunohistochemical analysis. Int. J. Immunopathol. Pharmacol. 2019, 33, 2058738419855226. [CrossRef]

36. Hasselbalch, I.C.; Søndergaard, H.B.; Koch-Henriksen, N.; Olsson, A.; Ullum, H.; Sellebjerg, F.; Oturai, A.B. The neutrophil-to-lymphocyte ratio is associated with multiple sclerosis. Mult. Scler. J. Exp. Transl. Clin. 2018, 4, 2055217318813183. [CrossRef]

37. Al-Hussain, F.; Alfallaj, M.M.; Alahmari, A.N.; Almazyad, A.N.; Alsaeed, T.K.; Abdurrahman, A.A.; Murtaza, G.; Bashir, S. Relationship between Neutrophil-to-Lymphocyte Ratio and Stress in Multiple Sclerosis Patients. J. Clin. Diagn. Res. 2017, 11, CC01-CC04. [CrossRef]

38. Fecchio, C.; Palazzi, L.; de Laureto, P.P. $\alpha$-Synuclein and Polyunsaturated Fatty Acids: Molecular Basis of the Interaction and Implication in Neurodegeneration. Molecules 2018, 23, 1531. [CrossRef]

39. Fedorova, M.; Bollineni, R.C.; Hoffmann, R. Protein carbonylation as a major hallmark of oxidative damage: Update of analytical strategies. Mass Spectrom. Rev. 2014, 33, 79-97. [CrossRef]

40. Suzuki, Y.J.; Carini, M.; Butterfield, D.A. Protein carbonylation. Antioxid. Redox Signal. 2010, 12, $323-325$. [CrossRef]

(C) 2019 by the authors. Licensee MDPI, Basel, Switzerland. This article is an open access article distributed under the terms and conditions of the Creative Commons Attribution (CC BY) license (http://creativecommons.org/licenses/by/4.0/). 